\title{
Rosiglitazone ameliorates astrocyte over-activation and inflammatory cytokine release induced by global cerebral ischemia/reperfusion
}

\author{
SHANLING REN, YAN XU, DONGWEI LV and LEI ZHANG \\ Department of Neurology, Zhongnan Hospital of Wuhan University, Wuhan, Hubei 430071, P.R. China
}

Received January 8, 2015; Accepted December 15, 2015

DOI: $10.3892 /$ etm.2016.2975

\begin{abstract}
Global cerebral ischemia (GCI) is a leading cause of mortality worldwide and remains the primary cause of long-term neurological disability. Astrocyte over-activation and extensive neuron loss in the ischemic brain are the characteristic pathological features of cerebral ischemia. Rosiglitazone (RSG) is a peroxisome-proliferating activating receptor- $\gamma$ agonist known for its anti-inflammatory activity. Previous studies have suggested that RSG is able to exert neuroprotection in numerous acute and chronic brain injury models. However, whether RSG treatment is involved in astrocyte over-activation and inflammatory reaction in the cortex remains unclear. The aim of the present study was to investigate whether RSG treatment improved functional impairment induced following GCI and protected against cortex neuron loss, and to elucidate the potential mechanisms underlying these functions. Rats were randomly divided into three groups: Sham-operated, GCI and RSG treatment groups. The RSG treatment group was treated with $2 \mathrm{mg} / \mathrm{kg} \mathrm{RSG}$ immediately following GCI. The results demonstrated that RSG treatment significantly reduced infarct volume and neuron survival rates in addition to increasing function recovery. Furthermore, these results correlate with a reduction in astrocyte over-activation and inflammatory cytokines in the rat cortex. However, no significant changes in glutamate transporter-1 expression levels were observed following RSG treatment compared with the GCI rats. The results of this investigation provide in vivo evidence that RSG significantly protected rats against ischemia-reperfusion-induced brain
\end{abstract}

Correspondence to: Dr Shanling Ren, Department of Neurology, Zhongnan Hospital of Wuhan University, 169 Donghu Road, Wuhan, Hubei 430071, P.R. China

E-mail: shanlingren24k@163.com

Abbreviations: RSG, rosiglitazone; GCI, global cerebral ischemia; $\mathrm{NeuN}$, neuron-specific nuclear protein; GFAP, glial fibrillary acidic protein; TNF- $\alpha$, tumor necrosis factor $\alpha$; IL- $1 \beta$, interleukin- $1 \beta$; IL-6, interleukin-6

Key words: rosiglitazone, global cerebral ischemia, inflammatory, astrocyte over-activation, glutamate transporter-1, rats injury. In addition, RSG may exert neuroprotective effects by inhibiting astrocyte over-activation, and thereby reducing the levels of inflammatory cytokines in the GCI-injured brain. All data revealed that RSG may be a potential neuroprotective agent for cerebral ischemia.

\section{Introduction}

Global cerebral ischemia (GCI), one of the consequences of surgical procedure and cardiac arrest, is a leading cause of disability and the second leading cause of mortality worldwide (1). Previous studies have revealed that intracellular calcium overload, oxidative stress, and post-ischemic glutamate and inflammatory cytokine release may be involved in the pathological process of GCI-induced brain injury (2-5). A complex interplay between various factors and signaling cascades leads to neuronal cell injury and death following ischemia (6,7). Although significant progress has been, made with extensive animal research into GCI treatment such as calcium channel blockers, radical scavengers, glutamate receptor antagonists and anti-inflammatory agents (7), few of these have been translated into clinically effective therapies (8).

As the most abundant cell type in the brain, astrocytes represent an attractive cellular candidate for GCI treatment (9). During the pathological process of GCI, astrocytes are able to protect neurons from injury via housekeeping mechanisms (9). Conversely, astrocytes are able to aggravate brain injury by releasing pro-inflammatory molecules and glutamate, thereby exacerbating the formation of brain edema $(10,11)$. However, few studies have investigated the effect of directly targeting astrocytes in the setting of GCI (10).

Rosiglitazone (RSG) is a peroxisome proliferating activating receptor- $\gamma$ (PPAR- $\gamma$ ) agonist known for its anti-inflammatory effects (12). Previous studies have demonstrated that treatment with RSG can exert neuroprotection in animal models of a number of conditions, including Alzheimer's disease, traumatic brain injury, spinal cord injury, and ischemia stroke (13-16). In addition, a recent study suggested that administration of RSG provided beneficial effects in the hippocampus of the rat brain following GCI (17); however, whether RSG treatment is involved in the astrocyte over-activation and inflammatory reaction in the 
cortex remains to be elucidated. Therefore, the present study aimed to investigate whether RSG treatment was able to improve functional impairment induced following GCI and protect against cortex neuron loss, in addition to elucidating the potential mechanisms underlying these functions.

\section{Materials and methods}

Animals and GCI model. A total of 180 rats adult female Sprague-Dawley rats (Wuhan University Animal Center, Wuhan, China), weighing 250-300 g and aged 3 months, were used in the present study. All procedures were approved by the Animal Care Welfare Committee of Zhongnan Hospital, Wuhan University for ethical experimentation on animals. All rats were provided with ad libitum access to food and water prior to the surgical procedure under optimal conditions (12-h light/dark cycle, $22^{\circ} \mathrm{C}$ ). Female rats were bilaterally ovariectomized, and 1 week later, GCI was induced by 4 -vessel occlusion as described previously (18). Briefly, the rats were anesthetized with $10 \%$ chloral hydrate (350 mg/kg, intraperitoneally; Beijing Solarbio Science \& Technology Co., Ltd., Beijing, China), the vertebral arteries were electrocauterized and the common carotid arteries (CCA) were exposed. Following $24 \mathrm{~h}$, the rats were anesthetized using $0.6 \mathrm{ml} / \mathrm{kg}$ isoflurane (intraperitoneally; Beijing Solarbio Science \& Technology Co., Ltd.) and the CCA were re-exposed and clipped using artery clips for $10 \mathrm{~min}$ followed by reperfusion. Rats that lost their righting reflex within $30 \mathrm{sec}$, those that had dilated pupils, and those that lost response to light during ischemia were selected for the experiments. Rectal temperature was maintained at $37 \pm 0.5^{\circ} \mathrm{C}$ using a thermal blanket during ischemia. Sham-operated animals underwent the same surgical procedures without occlusion of the CCA.

Group and drug administration. A total of 180 rats were randomly assigned to three groups: Sham-operated group (Sham, $n=60$ ); GCI group that received only equal volumes of $0.9 \%$ saline solution (GCI, $n=60)$; and a group treated with $2 \mathrm{mg} / \mathrm{kg}$ RSG (Cell Signaling Technology, Inc., Danvers, MA, USA) following GCI (RSG, n=60). RSG was dissolved in $0.9 \%$ saline and stored at $4^{\circ} \mathrm{C}$. Following GCI, RSG was immediately injected intraperitoneally in the rats of the RSG group following GCI $(2 \mathrm{mg} / \mathrm{kg})$. All tests were blinded, and the animal codes were revealed only at the end of the behavioral and histological analyses.

Measurement of the neurological deficit and infarct volume. Neurological deficit was evaluated $24 \mathrm{~h}$ following reperfusion according to the method described by Longa et al (18). Rats were anesthetized with $50 \mathrm{mg} / \mathrm{kg}$ sodium pentobarbital (i.p; Beijing Solarbio Science \& Technology Co., Ltd.) prior to sacrifice via exsanguination. The brains of the rats were then dissected and sectioned into five 2-mm coronal sections, that were incubated in 2\% 2,3,5-triphenyltetrazolium chloride (TTC; Amresco, LLC, Solon, OH, USA) for $15 \mathrm{~min}$ at $37^{\circ} \mathrm{C}$, as previously described (19) with minor modifications. The tissue sections were subsequently immersed and fixed in $4 \%$ paraformaldehyde (Beijing Solarbio Science \& Technology Co., Ltd.). The images of TTC-stained tissue sections were captured using an Olympus FE4000 digital camera (Olympus Corporation, Tokyo, Japan), and the digital images were analyzed using ImageJ image analysis software (version 1.41; National Institutes of Health, Bethesda, MA, USA). Infarct areas were measured and then compiled to obtain the infarct volume $\left(\mathrm{mm}^{3}\right)$ for each brain tissue sample.

Immunofluorescence. Coronal sections were incubated with $10 \%$ normal donkey serum for $30 \mathrm{~min}$ at room temperature in phosphate-buffered saline (PBS) and 0.1\% Triton X-100 (all Beijing Solarbio Science \& Technology Co., Ltd.) followed by incubation with appropriate primary antibodies overnight at $4^{\circ} \mathrm{C}$ in the same buffer. The frozen tissue sections were incubated with mouse anti-neuron-specific nuclear protein (NeuN) polyclonal antibody (1:100; sc-31154) and mouse anti-glial fibrillary acidic protein (GFAP) monoclonal immunoglobulin $(\mathrm{Ig}) \mathrm{G}_{1}$ (F7) antibody (1:100; sc-166458; both Santa Cruz Biotechnology, Inc., Dallas, TX, USA) overnight at $4^{\circ} \mathrm{C}$. The following day, the tissue sections were incubated with mouse FITC monoclonal $\operatorname{IgG}_{1}$ (1:1,000; sc-69871; Santa Cruz Biotechnology, Inc.) for $2 \mathrm{~h}$ at $37^{\circ} \mathrm{C}$ in the dark. Images were captured using a laser scanning confocal microscope (Olympus FV1000; Olympus Corporation, Tokyo, Japan). Primary antibodies were replaced with PBS in the negative control group.

Western blot analysis. Western blot analysis was conducted according to standard protocols (20). Rats were anesthetized using $50 \mathrm{mg} / \mathrm{kg}$ sodium pentobarbital and were intracardically perfused with $0.1 \mathrm{~mol} / 1 \mathrm{PBS}(\mathrm{pH}$ 7.4). The cortex region of the brain was rapidly isolated, homogenized (BestBio Biotechnology, Beijing, China), and total proteins were extracted using protein extraction reagent (Bio-Rad Laboratories, Inc., Hercules, CA, USA), according to manufacturer's protocol. Protein concentration was determined using a bicinchoninic acid assay (Beijing Solarbio Science $\&$ Technology Co., Ltd.). Briefly, $35 \mu \mathrm{g}$ total protein was separated by $20 \%$ SDS-polyacrylamide gel electrophoresis and electroblotted onto polyvinylidene fluoride membranes (EMD Millipore, Billerica, MA, USA) prior to being blocking with $5 \%$ fat-free dry milk for $1 \mathrm{~h}$ at room temperature. The membranes were subsequently incubated with the following primary antibodies overnight at $4^{\circ} \mathrm{C}$ : Rabbit anti-interleukin (IL)-1 $\beta$ polyclonal antibody (1:500; sc-7884), rabbit anti-IL-6 polyclonal antibody (1:500; sc-7920), rabbit anti-glutamate transporter (GLT)-1 polyclonal antibody (1:500; sc-15317), rabbit anti-tumor necrosis factor- $\alpha$ (TNF- $\alpha)$ polyclonal antibody (1:500; sc-7895), mouse anti- $\beta$-actin polyclonal antibody (1:500; sc-376421; all Santa Cruz Biotechnology, Inc.). Membranes were subsequently washed twice with Tris-buffered saline with Tween-20 (TBST) for $20 \mathrm{~min}$ and prior to incubation with horseradish peroxidase-conjugated anti-rabbit $\operatorname{IgG}(1: 5,000$; sc-2027) and anti-mouse $\operatorname{IgG}$ (1:5,000; sc-2025; both Santa Cruz Biotechnology, Inc.) for $2 \mathrm{~h}$ at room temperature. Membranes were washed four times with TBST for $40 \mathrm{~min}$. Protein bands on the membrane were visualized using an enhanced chemiluminescent reagent (EMD Millipore) and densitometric signals were quantified using ImageJ software (version 1.41; National Institutes of Health). 


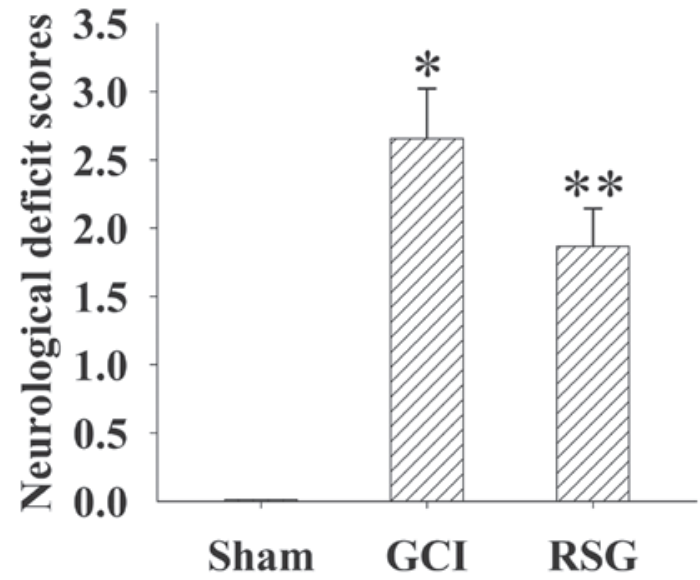

Figure 1. Effects of RSG on GCI-induced neurological deficits. Bars represent mean \pm standard error ( $n=5$ per group). GCI caused a significant increase in neurological deficits. Administration of RSG significantly attenuated neurological deficit scores. ${ }^{*} \mathrm{P}<0.01$ vs. the Sham group; ${ }^{* * *} \mathrm{P}<0.05$ vs. the GCI group. GCI, global cerebral ischemia; RSG, rosiglitazone.

A
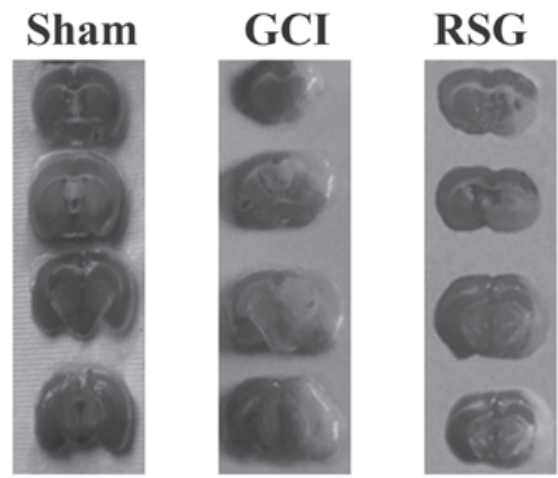

B

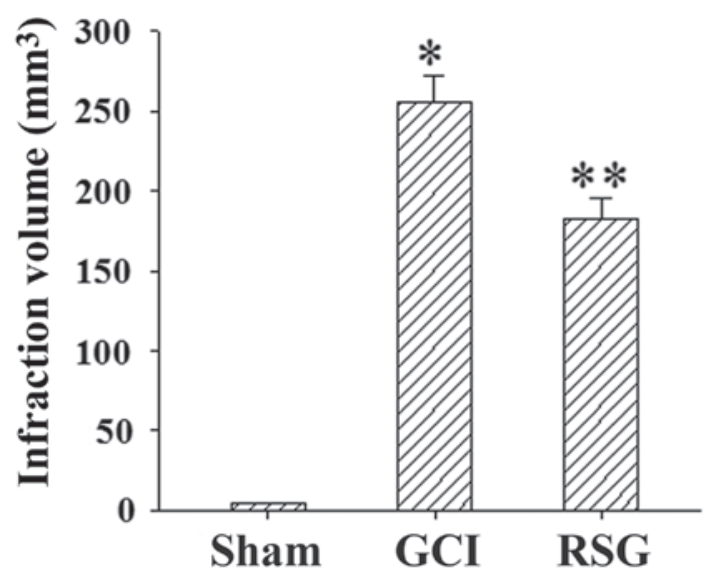

Figure 2. Effects of RSG on cerebral infarct volume. (A) TTC-stained brain sections showing the infarction area in three groups. (B) Bars represent mean \pm standard error ( $n=5$ per group). GCI caused a significant increase in cerebral infarct volume. RSG treatment decreased the cerebral infarct volume significantly. ${ }^{*} \mathrm{P}<0.01$ vs. the Sham group; ${ }^{* *} \mathrm{P}<0.05$ vs. the GCI group. GCI, global cerebral ischemia; RSG, rosiglitazone; TTC, 2,3,5-triphenyltetrazolium chloride.

Statistical analysis. All data were expressed as the mean \pm standard error of the mean. One way analysis of variance was used to assess statistical differences among the groups using SPSS 17.0 software (SPSS, Inc., Chicago, IL, USA). Significant
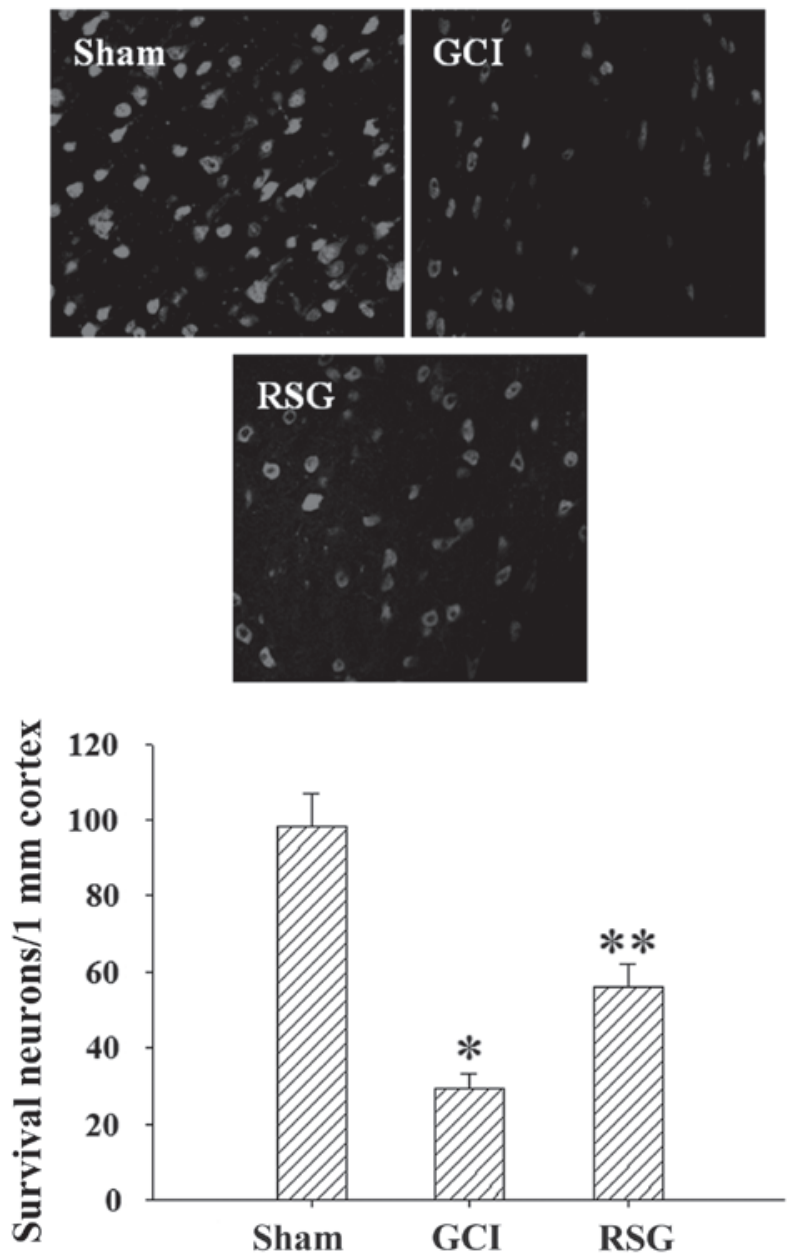

Figure 3. Effects of RSG on the neuronal survival in cortex tissue samples at $24 \mathrm{~h}$. Neuronal survival was observed using NeuN immunostaining (magnification, x400). Quantitative summary of the data shows the number of surviving neurons $/ 1 \mathrm{~mm}$. Bars represent mean \pm standard error $(\mathrm{n}=5$ per group). GCI caused a significant increase in neuron loss. The survival of neurons increased significantly following treatment with RSG. ${ }^{*} \mathrm{P}<0.01$ vs. the Sham group; ${ }^{* *} \mathrm{P}<0.01$ vs. the GCI group. GCI, global cerebral ischemia; RSG, rosiglitazone; NeuN, neuron-specific nuclear protein.

differences between groups at each time point were assessed by Student's t-test. $\mathrm{P}<0.05$ was considered to indicate a statistically significant difference.

\section{Results}

Treatment with RSG attenuates GCI-induced neurological deficits. Fig. 1 shows the changes in neurological deficit scores in the three groups. Post-injury administration of RSG significantly $(\mathrm{P}<0.05)$ improved neurological function recovery.

Treatment with RSG attenuates cerebral infarct volume. Ischemia/reperfusion produced marked infarction, as demonstrated in the serial coronal brain tissue sections (Fig. 2A). RSG treatment significantly $(\mathrm{P}<0.05)$ reduced the infarct volume, as compared with the GCI group at $24 \mathrm{~h}$ post-GCI (Fig. 2B).

Treatment with RSG suppresses neuronal death in the cortex following GCI. The neuronal survival rate in the rat cortex 

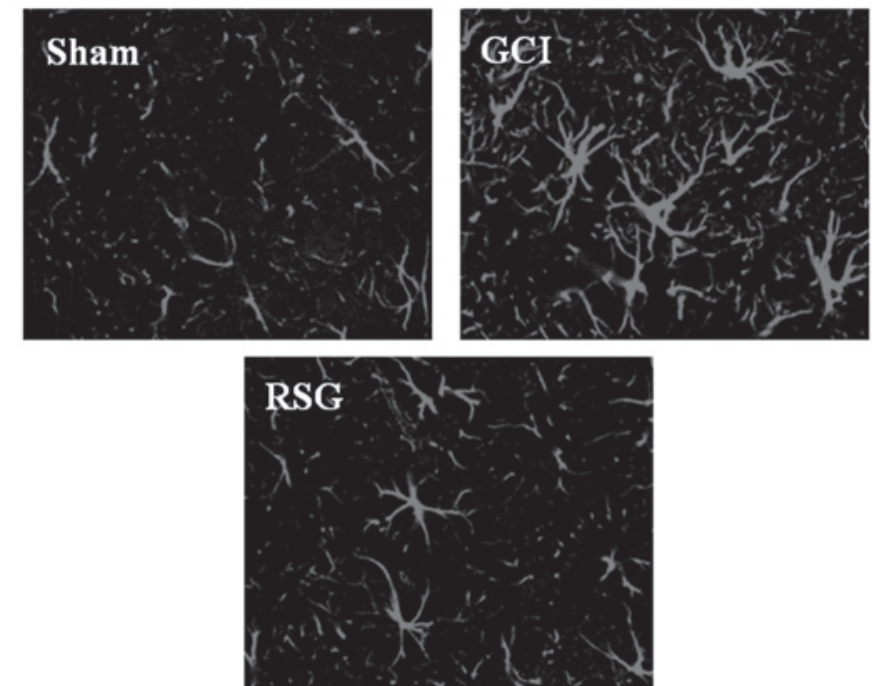

Figure 4. Astrocyte activation was observed using GFAP immunostaining ( $\mathrm{n}=5$ per group) (magnification, $\mathrm{x} 400$ ). GCI caused marked astrocyte activation compared with the Sham group. Astrocyte activation significantly reduced in the RSG treatment group compared with the GCI group. GCI, global cerebral ischemia; RSG, rosiglitazone; GFAP, glial fibrillary acidic protein.

$\mathbf{A}$

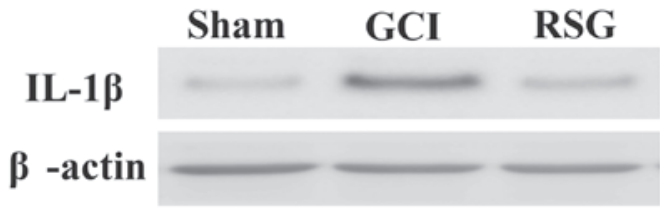

B

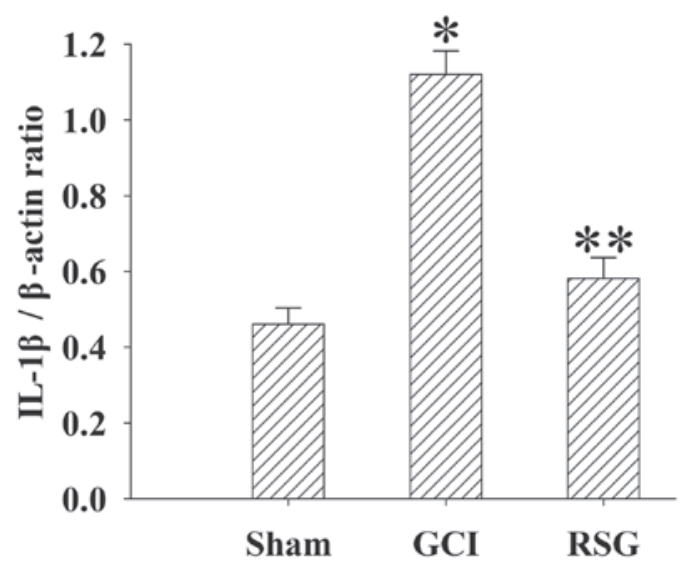

Figure 5. (A) Western blot analysis shows the expression of IL-1 $\beta$ in the cortex tissue samples of the three groups. (B) The quantitative results of IL- $1 \beta$ were expressed as the ratio of IL-1 $\beta$ to $\beta$-actin band density. Means \pm standard error ( $\mathrm{n}=5$ per group). ${ }^{*} \mathrm{P}<0.01$ vs. the Sham group; ${ }^{* *} \mathrm{P}<0.01$ vs. the GCI group. GCI, global cerebral ischemia; RSG, rosiglitazone; IL-1 $\beta$, interleukin-1 $\beta$.

was assessed using anti-NeuN antibody at $24 \mathrm{~h}$ post-GCI. As shown in Fig. 3, GCI caused significant $(\mathrm{P}<0.01)$ neuron loss compared with the Sham group; however, this effect was partly reversed by treatment with RSG.

Treatment with RSG attenuates astrocyte over-activation induced by GCI. It was next examined whether RSG treatment affected astrocytes in the rat cortex by using anti-GFAP antibodies as a marker for activated astrocytes. As shown in Fig. 4, GCI induced marked astrocyte over-activation.
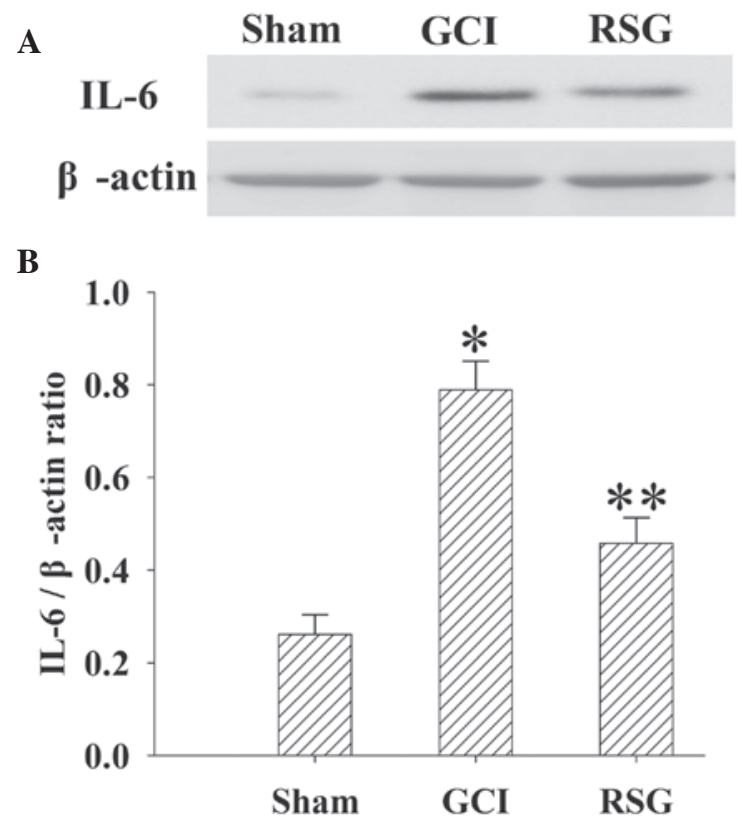

Figure 6. (A) Western blot analysis shows the expression levels of IL-6 in the cortex of the three groups. (B) The quantitative results of IL-6 were expressed as the ratio of IL-6 to $\beta$-actin band density. Means \pm standard error ( $n=5$ per group). ${ }^{*} \mathrm{P}<0.01$ vs. the Sham group; ${ }^{* *} \mathrm{P}<0.01$ vs. the GCI group. GCI, global cerebral ischemia; RSG, rosiglitazone; IL-6, interleukin-6.

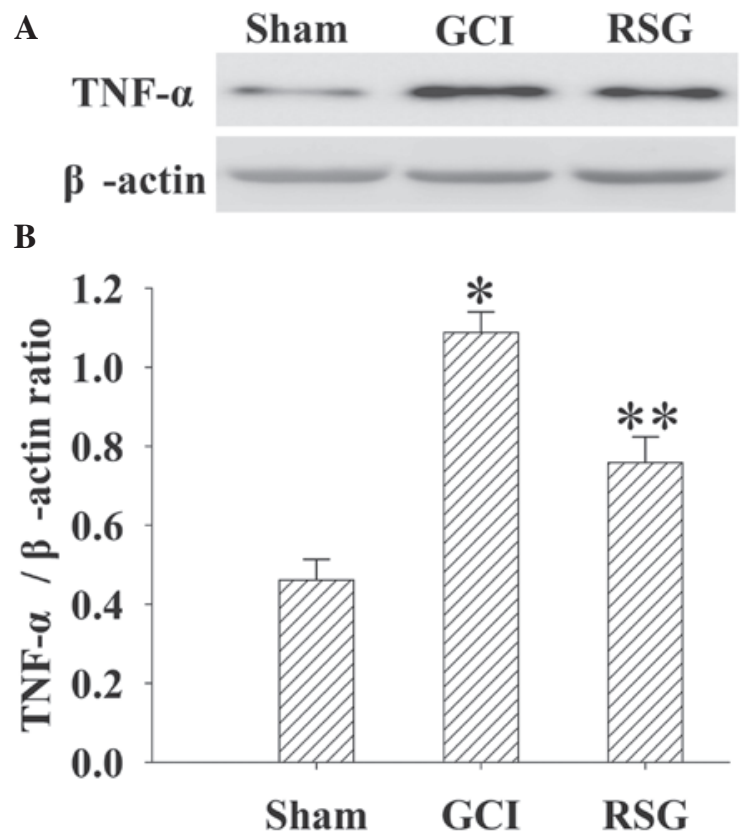

Figure 7. (A) Western blot analysis shows the expression of TNF- $\alpha$ in the cortex of the three groups. (B) The quantitative results of TNF- $\alpha$ were expressed as the ratio of TNF- $\alpha$ to $\beta$-actin band density. Means \pm standard error ( $n=5$ per group). ${ }^{*} \mathrm{P}<0.01$ vs. the Sham group; ${ }^{* *} \mathrm{P}<0.01$ vs. the GCI group. GCI, global cerebral ischemia; RSG, rosiglitazone; TNF- $\alpha$, tumor necrosis factor.

However, RSG caused a marked attenuation of astrocyte activation compared with the GCI group.

Treatment with $R S G$ attenuates $I L-1 \beta$ expression in the rat cortex following GCI. The expression levels of IL-1 $\beta$ in the cortex at $24 \mathrm{~h}$ were measured by western blotting (Fig. 5). 


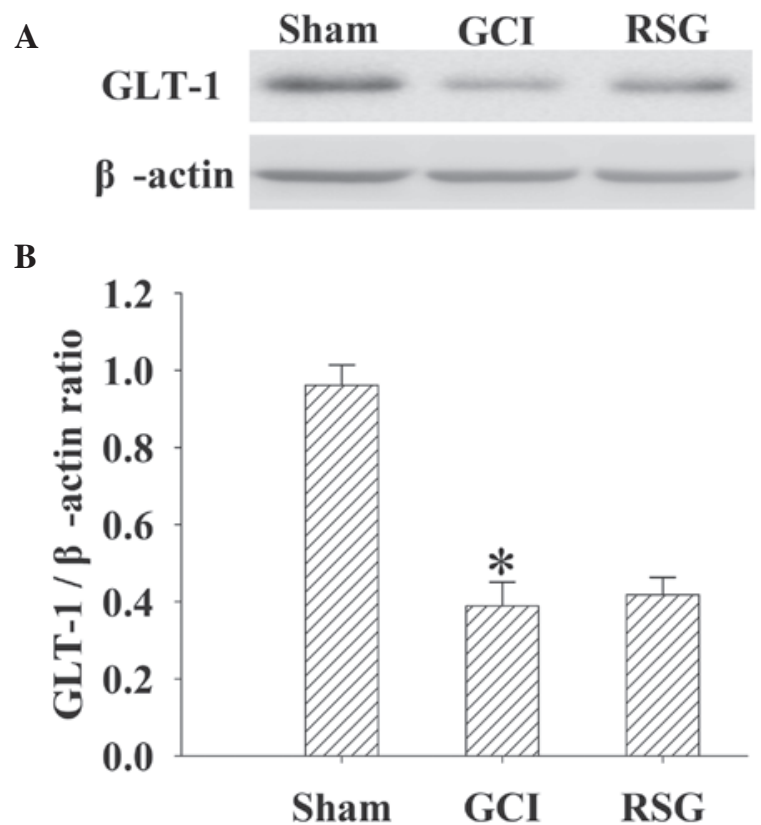

Figure 8. (A) Western blot analysis shows the expression of GLT-1 in the cortex brain tissue samples of the three groups. (B) The quantitative results of GLT- 1 were expressed as the ratio of GLT- 1 to $\beta$-actin band density. Means \pm standard error $(n=5)$. A significantly downregulation in GLT-1 expression levels were observed in the GCI group. ${ }^{*} \mathrm{P}<0.01$ vs. the Sham group. RSG caused on significantly changes of GLT-1 expression. GCI, global cerebral ischemia; RSG, rosiglitazone; GLT-1, glutamate transporter-1.

IL-1 $\beta$ expression levels were significantly elevated in the GCI group, as compared with the Sham group. However, treatment with RSG induced a significant $(\mathrm{P}<0.01)$ reduction in IL-1 $\beta$ expression levels.

Treatment with RSG attenuates IL-6 expression levels in the cortex following GCI. The expression levels of IL-6 in the cortex at $24 \mathrm{~h}$ were measured by western blotting (Fig. 6). IL-6 expression levels were significantly increased $(\mathrm{P}<0.01)$ in the GCI group, as compared with the Sham group. However, treatment with RSG significantly $(\mathrm{P}<0.01)$ decreased IL-6 expression levels, as compared with the GCI group.

Treatment with RSG attenuates TNF- $\alpha$ expression levels in the cortex following GCI. The expression levels of TNF- $\alpha$ in the cortex at $24 \mathrm{~h}$ were measured by western blotting (Fig. 7). TNF- $\alpha$ expression levels were significantly $(\mathrm{P}<0.01)$ elevated in the GCI group, as compared with the Sham group. However, administration of RSG induced a significant $(\mathrm{P}<0.01)$ reduction in the GCI-induced upregulation of TNF- $\alpha$ expression levels.

No significant changes in GLT-1 expression levels were observed in the cortex tissue samples following RSG treatment. GLT-1 protein expression levels in the cortex were analyzed by western blot analysis at $24 \mathrm{~h}$ (Fig. 8). Significant $(\mathrm{P}<0.01)$ downregulation in GLT-1 expression levels was detected in the GCI group, as compared with the Sham group. However, treatment with RSG induced no significant $(\mathrm{P}>0.05)$ changes in GLT-1 expression levels.

\section{Discussion}

GCI is a leading cause of mortality worldwide and remains the primary cause of long-term neurological disability (21). Astrocyte over-activation as well as extensive loss of neurons in the ischemic brain are the characteristic pathological features of ischemia stroke (22). One reason for the failure to translate successful results in animal experiments to clinical therapies may be due to the complexity of signaling responses which reduce the likelihood that altering a single target will be effective $(6,7)$. The present study investigated the efficacy of the PPAR- $\gamma$ agonist, RSG, as a therapeutic strategy for the treatment of GCI. The results demonstrated that RSG treatment immediately following GCI significantly reduced infarct volume and neuron survival rate, in addition to increasing functional recovery. Furthermore, these results correlated with a reduction in astrocyte over-activation and inflammatory cytokines in the rat cortex. Previous studies have demonstrated that RSG provides neuroprotective effects in numerous acute and chronic brain injury models, including amyotrophic lateral sclerosis, Alzheimer's disease, traumatic brain injury, spinal cord injury and ischemia stroke (13-16). The data of the present study were concordant with those of previous investigations, and to the best of our knowledge reported for the first time that post-GCI administration of RSG provided neuroprotective effects via attenuation of astrocyte over-activation in the cortex.

An important delayed mechanism beginning within hours of the onset of GCI-induced brain injury is the inflammatory response in the ischemic tissue (22). In particular, cerebral ischemia rapidly elevated inflammatory responses in the rat brain, thereby contributing to blood brain barrier disruption and delayed neuronal death $(5,22)$. Therefore, therapeutic strategies targeting the delayed inflammatory response may inhibit the progression of the tissue damage, which would provide an extended therapeutic window for neuroprotection on GCI. However, during the response to ischemic injury, microglia and astrocytes are activated in the brain (23). Astrocytes are sensitive to the increased release of these immunomodulatory peptides and therefore severe ischemia also compromises astrocytic function $(23,24)$. Following GCI, astrocytes rapidly become over-activated and undergo morphological transformations, accompanied by functional changes such as increasing expression levels of cytokines, including TNF- $\alpha$, interleukins (IL-1 $\beta$, IL-4, IL-6, IL-10), chemokines and interferons (25). Subsequently, the accumulation of pro-inflammatory factors further exacerbates ischemic damage (26-28). In the present study, the result demonstrated that ischemic injury resulted in over-activation of astrocytes, and thereby significantly elevated the expression levels of IL-1 $\beta$, IL- 6 , and TNF- $\alpha$ in the cortex. Furthermore, it is worth noting that treatment with RSG was able to inhibit astrocyte over-activation, and reduce the levels of these inflammatory factors. Although the role of anti-inflammatory factors in stroke patients remains to be fully elucidated, the majority of the existing studies have demonstrated that RSG is able to exert neuroprotection via its anti-inflammatory activity in numerous animal models of neurological disorders (13-16). Therefore, the results herein suggest that RSG exerts its neuroprotective effects by 
inhibiting astrocyte over-activation, and thereby attenuating inflammatory cytokine release.

Astrocytes protect against glutamate excitotoxicity via glutamate transporters, and GLT-1 is responsible for $\sim 90 \%$ of all glutamate transport in adult brain tissue (29). Previous studies have demonstrated that Ceftriaxone treatment, which induces astrocytic glutamate uptake via elevated GLT-1, exerted neuroprotection effects in numerous models of neurological disease (30-32). Similarly, selective overexpression of GLT-1 in astrocytes also provided neuroprotection following focal or global cerebral ischemia (33). However, in the present study, no significant changes in GLT-1 expression levels were observed following RSG treatment compared with the GCI rats, which suggested that the neuroprotective effect of RSG does not appear to be mediated by the modulation of GLT-1 protein expression levels in the rat model of GCI.

In conclusion, the present investigation demonstrated that RSG significantly protected rats against ischemia-reperfusion-induced brain injury. In addition, RSG may exert neuroprotective effects by inhibiting astrocyte over-activation, and thereby reduces the levels of inflammatory cytokines in the GCI-injured brain.

\section{Acknowledgements}

The present study was supported by a grant from the Nature Science Foundation of Hubei Province (grant no. 2014CFB479).

\section{References}

1. Cao Y, Mao X, Sun C, Zheng P, Gao J, Wang X, Min D, Sun H, Xie N and Cai J: Baicalin attenuates global cerebral ischemia/reperfusion injury in gerbils via anti-oxidative and anti-apoptotic pathways. Brain Res Bull 85: 396-402, 2011.

2. Kristián T and Siesjö BK: Calcium in ischemic cell death. Stroke 29: 705-718, 1998.

3. Love S: Oxidative stress in brain ischemia. Brain Pathol 9: 119-131, 1999.

4. Choi DW and Rothman SM: The role of glutamate neurotoxicity in hypoxic-ischemic neuronal death. Annu Rev Neurosci 13: 171-182, 1990.

5. Huang J, Upadhyay UM and Tamargo RJ: Inflammation in stroke and focal cerebral ischemia. Surg Neurol 66: 232-245, 2006.

6. Shamloo M, Rytter A and Wieloch T: Activation of the extracellular signal-regulated protein kinase cascade in the hippocampal CA1 region in a rat model of global cerebral ischemic preconditioning. Neuroscience 93: 81-88, 1999.

7. Mehta SL, Manhas N and Raghubir R: Molecular targets in cerebral ischemia for developing novel therapeutics. Brain Res Rev 54: 34-66, 2007.

8. Liu S, Levine SR and Winn HR: Targeting ischemic penumbra: Part I-from pathophysiology to therapeutic strategy. J Exp Stroke Transl Med 3: 47-55, 2010.

9. Ogata K and Kosaka T: Structural and quantitative analysis of astrocytes in the mouse hippocampus. Neuroscience 113: 221-233, 2002.

10. Pekny $\mathbf{M}$ and Nilsson $\mathbf{M}$ : Astrocyte activation and reactive gliosis. Glia 50: 427-434, 2005.

11. Swanson RA, Ying W and Kauppinen TM: Astrocyte influences on ischemic neuronal death. Curr Mol Med 4: 193-205, 2004.

12. Mohanty P, Aljada A, Ghanim H, Hofmeyer D, Tripathy D, Syed T, Al-Haddad W, Dhindsa S and Dandona P: Evidence for a potent antiinflammatory effect of rosiglitazone. J Clin Endocrinol Metab 89: 2728-2735, 2004.
13. Escribano L, Simón A-M, Pérez-Mediavilla A, Salazar-Colocho P, Del Río J and Frechilla D: Rosiglitazone reverses memory decline and hippocampal glucocorticoid receptor down-regulation in an Alzheimer's disease mouse model. Biochem Biophys Res Commun 379: 406-410, 2009.

14. Yi JH, Park SW, Brooks N, Lang BT and Vemuganti R: PPARgamma agonist rosiglitazone is neuroprotective after traumatic brain injury via anti-inflammatory and anti-oxidative mechanisms. Brain Res 1244: 164-172, 2008.

15. Zhang Q, Hu W, Meng B and Tang T: PPAR $\gamma$ agonist rosiglitazone is neuroprotective after traumatic spinal cord injury via anti-inflammatory in adult rats. Neurol Res 32: 852-859, 2010.

16. Luo Y, Yin W, Signore AP, Zhang F, Hong Z, Wang S, Graham SH and Chen J: Neuroprotection against focal ischemic brain injury by the peroxisome proliferator-activated receptor-gamma agonist rosiglitazone. J Neurochem 97: 435-448, 2006.

17. Al Rouq F and El Eter E: PPAR- $\gamma$ activator induces neuroprotection in hypercholesterolemic rats subjected to global cerebral ischemia/reperfusion injury: In vivo and in vitro inhibition of oxidative stress. Exp Gerontol 51: 1-7, 2014.

18. Longa EZ, Weinstein PR, Carlson S and Cummins R: Reversible middle cerebral artery occlusion without craniectomy in rats. Stroke 20: 84-91, 1989.

19. Bederson JB, Pitts LH, Germano SM, Nishimura MC, Davis RL and Bartkowski HM: Evaluation of 2,3,5-triphenyltetrazolium chloride as a stain for detection and quantification of experimental cerebral infarction in rats. Stroke 17: 1304-1308, 1986.

20. Shimamura N, Matchett G, Solaroglu I, Tsubokawa T, Ohkuma H and Zhang J: Inhibition of integrin $\alpha$ vbeta3 reduces blood-brain barrier breakdown in focal ischemia in rats. J Neurosci Res 84: 1837-1847, 2006.

21. Strong K, Mathers C and Bonita R: Preventing stroke: Saving lives around the world. Lancet Neurol 6: 182-187, 2007.

22. Vexler ZS, Tang XN and Yenari MA: Inflammation in adult and neonatal stroke. Clin Neurosci Res 6: 293-313, 2006.

23. Willis CL: Glia-induced reversible disruption of blood-brain barrier integrity and neuropathological response of the neurovascular unit. Toxicol Pathol 39: 172-185, 2011.

24. Wang Q, Tang XN and Yenari MA: The inflammatory response in stroke. J Neuroimmunol 184: 53-68, 2007.

25. Pickering $\mathrm{M}$ and O'Connor JJ: Pro-inflammatory cytokines and their effects in the dentate gyrus. Prog Brain Res 163: 339-354, 2007.

26. Kaushal V and Schlichter LC: Mechanisms of microglia-mediated neurotoxicity in a new model of the stroke penumbra. J Neurosci 28: 2221-2230, 2008.

27. Barone FC and Parsons AA: Therapeutic potential of anti-inflammatory drugs in focal stroke. Expert Opin Investig Drugs 9: 2281-2306, 2000.

28. Batti L and O'Connor JJ: Tumor necrosis factor-alpha impairs the recovery of synaptic transmission from hypoxia in rat hippocampal slices. J Neuroimmunol 218: 21-27, 2010.

29. Anderson CM and Swanson RA: Astrocyte glutamate transport: Review of properties, regulation and physiological functions. Glia 32: 1-14, 2000.

30. Chu K, Lee ST, Sinn DI, Ko SY, Kim EH, Kim JM, Kim SJ, Park DK, Jung KH, Song EC, et al: Pharmacological Induction of ischemic tolerance by glutamate transporter-1 (EAAT2) upregulation. Stroke 38: 177-182, 2007.

31. Wei J, Pan X, Pei Z, Wang W, Qiu W, Shi Z and Xiao G: The beta-lactam antibiotic, ceftriaxone, provides neuroprotective potential via anti-excitotoxicity and anti-inflammation response in a rat model of traumatic brain injury. $\mathrm{J}$ Trauma Acute Care Surg 73: 654-660, 2012.

32. Azbill RD, Mu X and Springer JE: Riluzole increases high-affinity glutamate uptake in rat spinal cord synaptosomes. Brain Res 871: 175-180, 2000.

33. Harvey BK, Airavaara M, Hinzman J, Wires EM, Chiocco MJ, Howard DB, Shen H, Gerhardt G, Hoffer BJ and Wang Y: Targeted over-expression of glutamate transporter 1 (GLT-1) reduces ischemic brain injury in a rat model of stroke. PLoS One 6: e22135, 2011. 